\title{
Research on the Random Shock Vibration Test Based on the Filter-X LMS Adaptive Inverse Control Algorithm
}

\author{
Wei Wang, Zhangwei Chen, Qiangli Luan \\ The State Key Lab of Fluid Power \& Mechatronic Systems, Zhejiang University, Hangzhou 310013, China
}

\begin{abstract}
The related theory and algorithm of adaptive inverse control were presented through the research which pointed out the adaptive inverse control strategy could effectively eliminate the noise influence on the system control. Proposed using a frequency domain filter-X LMS adaptive inverse control algorithm, and the control algorithm was applied to the two-exciter hydraulic vibration test system of random shock vibration control process and summarized the process of the adaptive inverse control strategies in the realization of the random shock vibration test. The self-closed-loop and field test show that using the frequency-domain filter-X LMS adaptive inverse control algorithm can realize high precision control of random shock vibration test.
\end{abstract}

\section{Introduction}

Shock test [1]-[3] is mainly used in the simulation of the transient impact signal of falling, shelling and artillery firing as a common vibration test method. Since in the process of the real shock (such as the missile launch), the shock signal on the specimen is not standard combined with stochastic and shock characteristics at the same time, so the random shock vibration test is put forward, which can simulate the environment shock load on specimen.

Effective control of multi-axis hydraulic vibration test system relies on the accurate estimation of system frequency-response function (FRF) matrix, since the existence of nonlinear factors in the system, lead to the error between the actual system FRF matrix and the control matrix (FRF), to eliminate this error, there must be real-time update of the system FRF matrix to reduce the error between the control matrix and the actual matrix. This paper proposed using a frequency-domain filter-X LMS adaptive inverse control algorithm, and applied to the random shock vibration control process of two-exciter hydraulic vibration test system and summarize the process of the realization of adaptive inverse control strategies. The self-closed-loop and field test show that using the frequency-domain filter-X LMS adaptive inverse control algorithm can realize high precision control of random shock vibration test.

\section{Adaptive inverse control strategy}

Adaptive inverse control technology [4], [5] based on the effective control of inverse transfer function of controlled object, to realize the rapid and accurate tracking of the instructions from output to input. The reference model $M(f)$ is the requirements of control system, means the delay link, which can ensure that the stability of the control of delay system and non-minimum phase system, and ensure the controller has enough control precision to realize the precise and small error response output of control system. The transfer function $H(f)$, the inverse transfer function of controlled objects (impedance function) $Z(f)_{k}\{k=0 \cdots \cdots \mathrm{N}\}$ as the transfer function of the controller, mutual relations are represented as:

$$
Z(f)=\frac{M(f)}{H(f)}
$$

For adaptive inverse control system, external interference signal will result in the divergence of solution of system impedance function which means the formula (1) is not satisfied. To eliminate the influence of noise interference on the system and consider the effective control of the system using adaptive LMS algorithm, the paper puts forward a frequency domain of filter-X LMS adaptive inverse control algorithm, and apply it to the random shock vibration control process of two-exciter hydraulic vibration test system, the control process mainly includes the object modeling, inverse modeling and adaptive inverse control.

Object modeling $H_{v}$ method was adopted to realize the initial transfer function recognition, to improve the precision of the system transfer function identification, the process of multi-times identification and average processing are conducted, until the precision meet the requirements. Frequency filter-X LMS adaptive inverse control algorithm does not affect the Wiener solution of controller, even the object identification model error exists. 
Inverse modeling process using the copied object model $\hat{H}(f)$ to realize the identification of the system impedance function $Z(f)$ with adaptive LMS algorithm.

Adaptive inverse control process will regard $Z(f)$ as the controller transfer function which is applied to the actual physical model $H(f)$ to achieve effective control.

\section{Random shock vibration control}

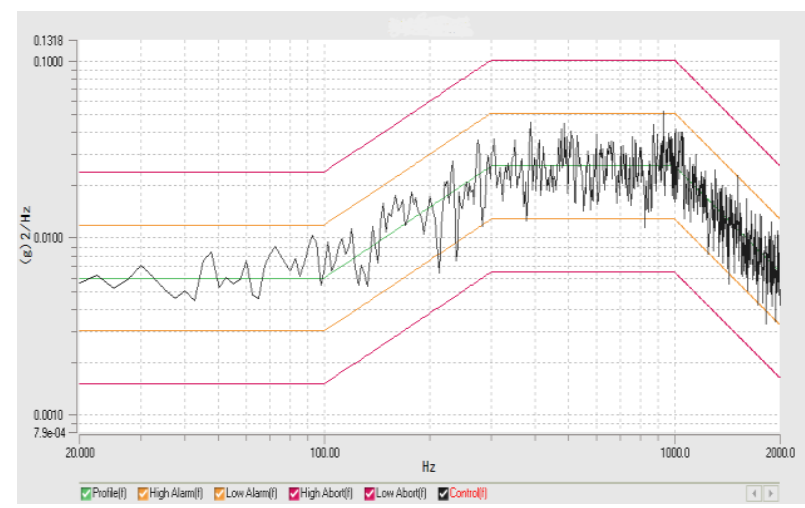

(a) random signal power spectrum density function

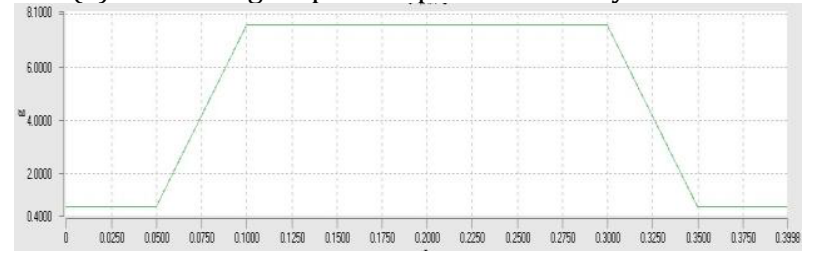

(b) random signal cutting waveform

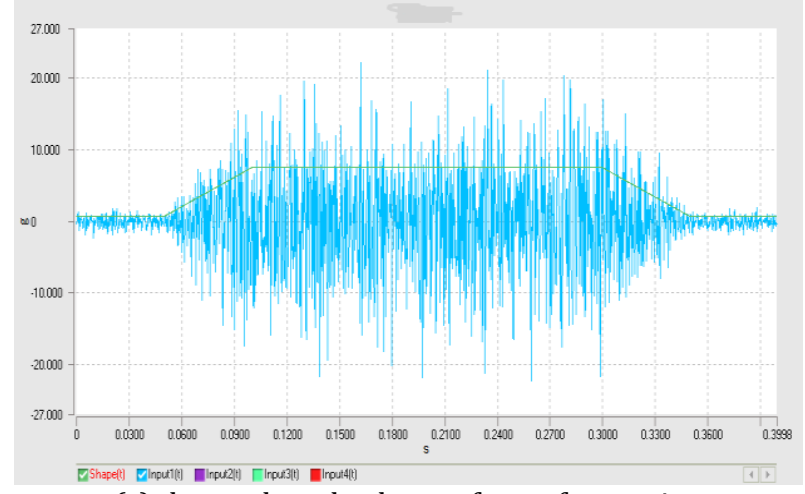

(c) the random shock waveform after cutting

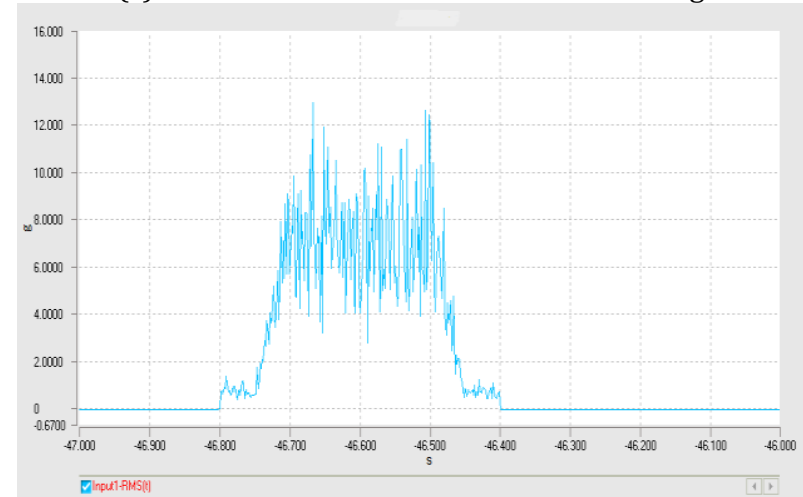

(d) the random shock waveform RMS after cutting

Figure 1. random shock vibration teat waveform.

In random shock vibration test [6], set the random signal power spectrum density function firstly (1 (a)), the reference spectrum generate driving signal power spectrum reiteration according to the test process, after cutting by window (1(b), then input to the vibration test system, the response (control) signal(1(c)), control signal effective value (1(d)).

\section{Test system}

In this paper, the control core of random shock hydraulic vibration test system is the Premax VT - 6008 servo vibration controller ${ }^{[7]}$ which is based on a frequency domain filter-X LMS adaptive inverse control algorithm provided by Hangzhou ECON Technology co., LTD, it adopt integrated PID control and double closed-loop control, the control frequency range can be up to $0.1 \mathrm{~Hz}$ $400 \mathrm{~Hz}$ and applicable to the vertical or horizontal single degree of freedom test and tri-axial six degrees of freedom test; the test subject is the two-exciter hydraulic vibration test system provided by the State Key Lab of Fluid Power \& Mechatronic Systems of Zhejiang university.

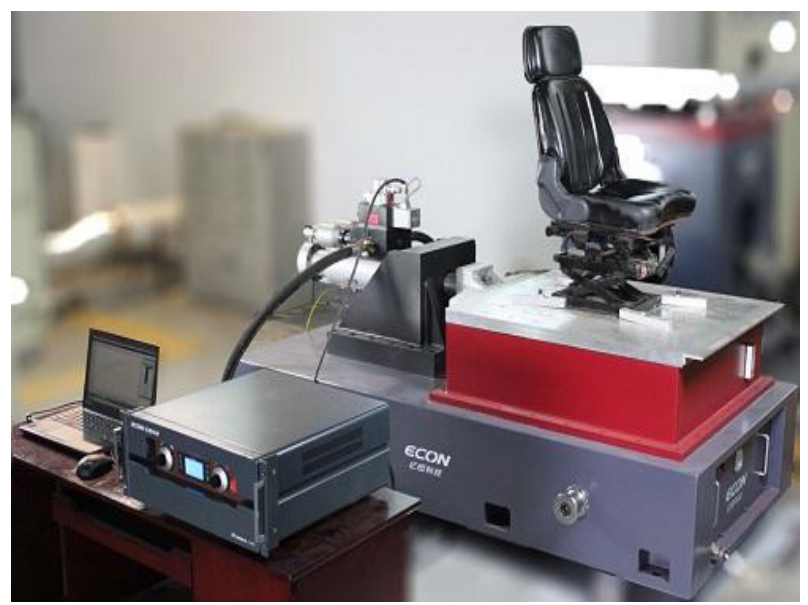

Figure 2. Premax VT - 6008 servo vibration controller

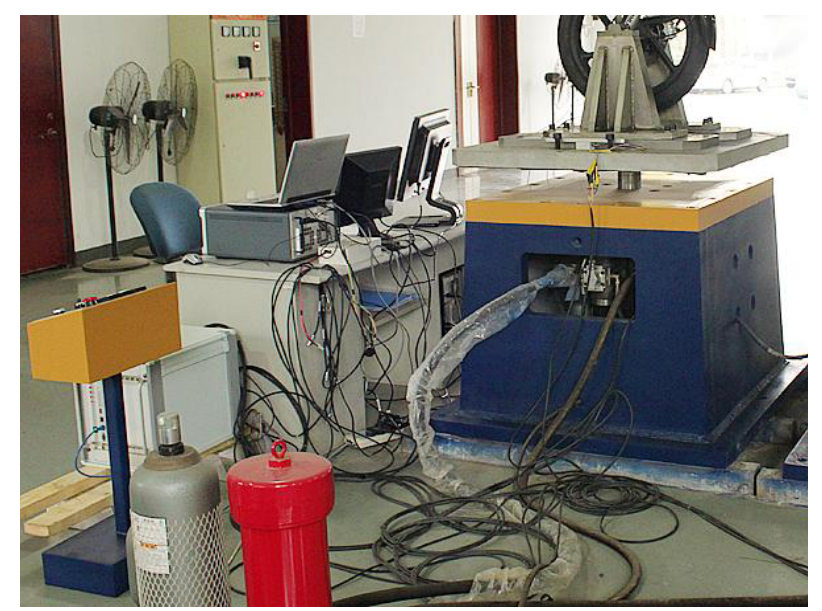

Figure 3. the two-exciter hydraulic shock vibration test system

\section{Random vibration test}

\subsection{The self-closed-loop test}


The closed-loop test chose 2 output (incentives) channels, 2 input (control) channels. The bandwidth of random power spectral is $5 \sim 100 \mathrm{~Hz}$, the control signal effective value is $0.15 \mathrm{~g}$, the correlation coefficient between signals is 0.2. Fig. 4(a) $\sim$ (d) for show the closed-loop test system results: Figure 4(a), 5(b) show the 2 input (control) the power spectral density curve of spectrum (control) after the signal energy recovery; Fig. 4 (c), 4 (d) show the 2 input (control) in the time domain waveform curve of the signal. According to the results of the closed-loop test, the control spectrum curve can repeat the reference spectrum waveform in high precision, at the same time, the input waveform can realize the expected random shock waveform according to the shape spectrum (Fig. 1).

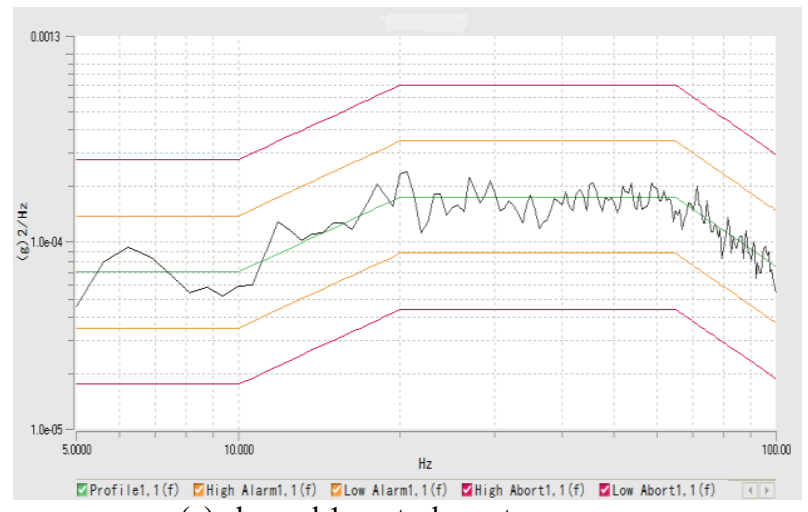

(a) channel 1 control spectrum

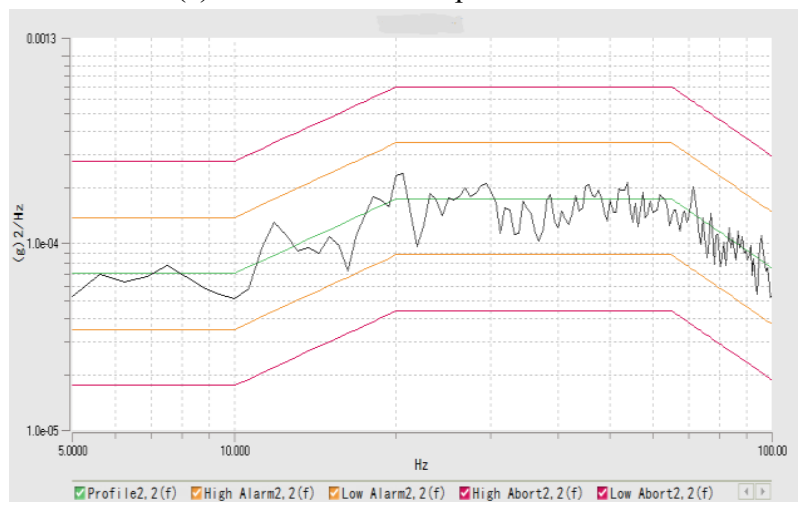

(b) channel 2 control spectrum

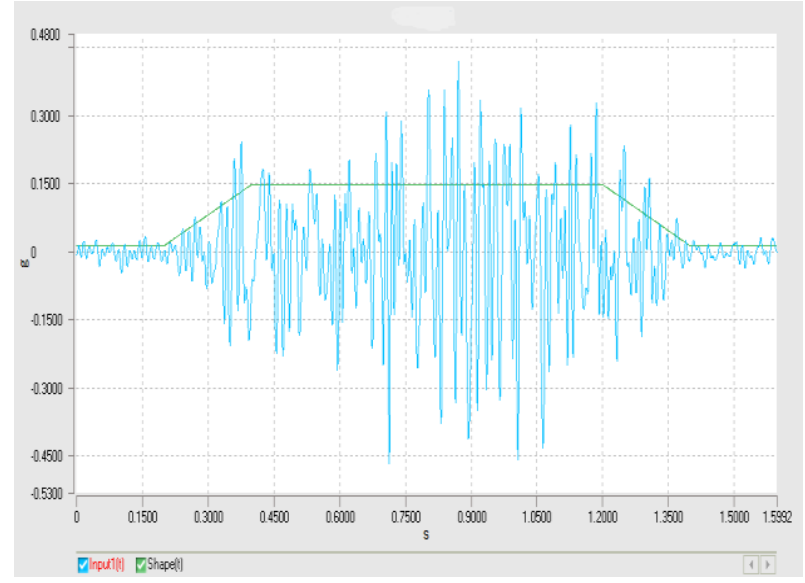

(c) channel 1 control signal

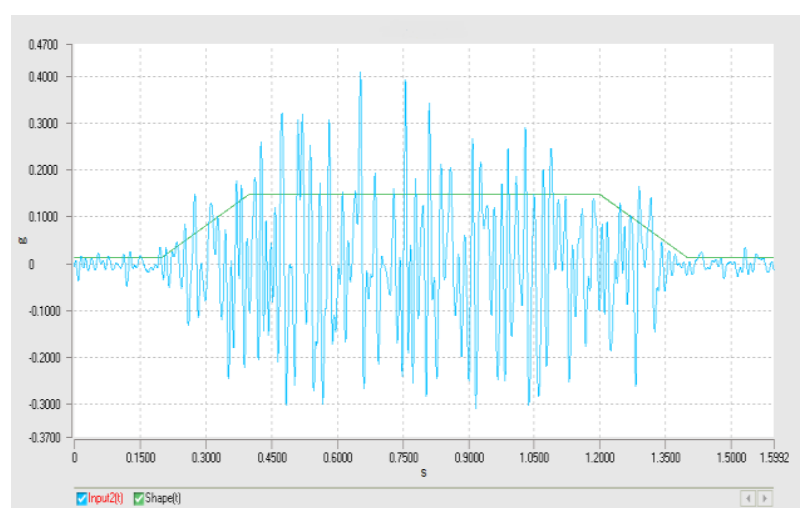

(d) channel 2 control signal

Figure 4. the result of random shock closed-loop test

The closed-loop test shows that the random shock test based on the frequency domain filter-X LMS adaptive inverse control algorithm can achieve high precision in the self-close-loop control, the spectrum control precision satisfies the requirement of experiment.

\subsection{The field test}

The random shock vibration test on the two-exciter hydraulic shock vibration test system for is shown in Fig. 5 in 2 points incentive, 2 point control experiment, the field test reference spectrum is the same to the closed-loop test. After 4 effective iterative calculation, the random shock vibration test of the two-exciter hydraulic shock vibration test system show in Fig. 6 (a) (d). Fig. 6 (a) and (b) are the control signal (after energy recovery) power spectral density curve, Fig. 6 (c) and (d) are the control signal time domain waveform curve.

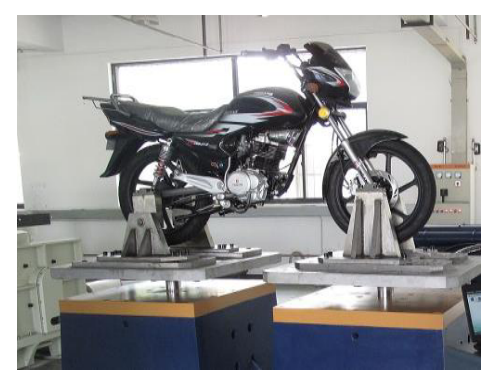

Figure 5 the two-exciter hydraulic shock vibration test system

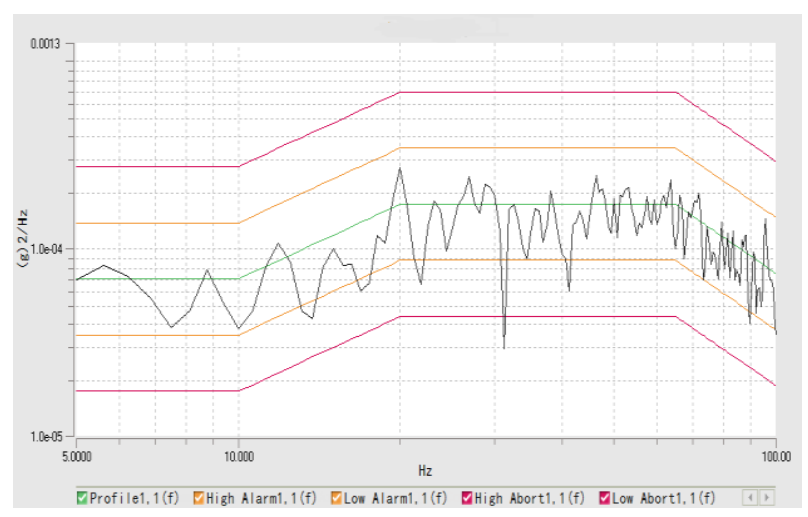

(a) channel 1 control spectrum 


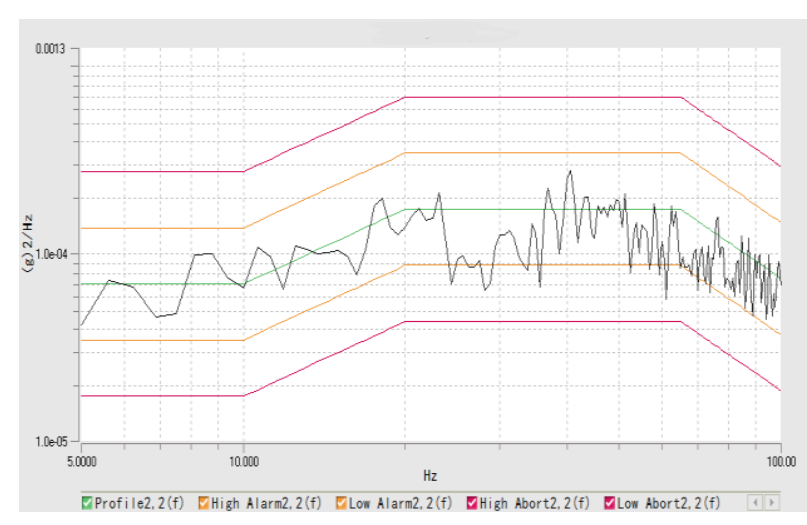

(b) channel 2 control spectrum

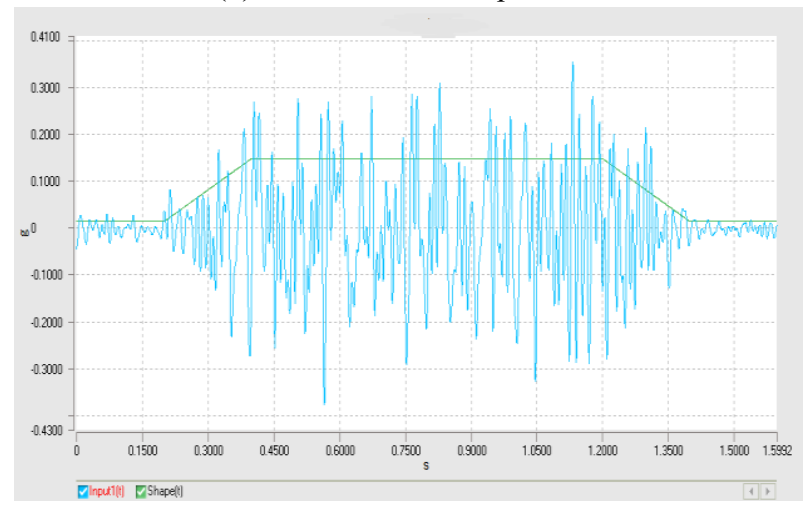

(c) channel 1 control signals

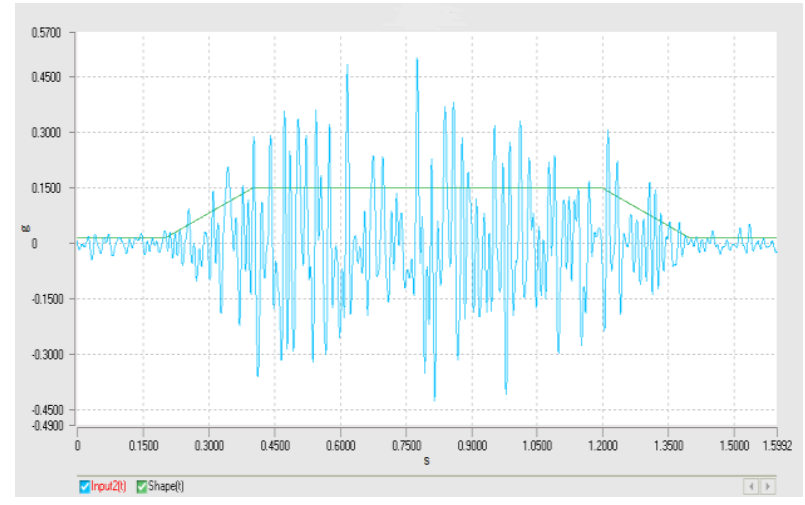

(d) channel 2 control signals

Figure 6. results of the random shock field tests

From the random shock vibration test on the two-exciter hydraulic shock vibration test system, within the scope of the test frequency width $(5 \mathrm{~Hz} \sim 100 \mathrm{~Hz})$, the scope of the error of the power spectral density curve of the control signal is control within $\pm 1.5 \mathrm{~dB}$ ( as shown in Fig. 6 (a) and (b)), as the test progressed and the increase of iterations, the field test can achieve good control effect, the random shock test can control time domain signal according to the cutting waveform. Further, the test can realize the high precision control by using the frequency domain filter-X LMS adaptive inverse control strategy, thus proving the effectiveness of the proposed control strategy.

\section{Conclusion}

This paper adopts the two-exciter hydraulic shock vibration test system as the test object and Premax VT 6008 servo controller as the control core to conduct the vibration test research based on filter-X frequency domain LMS adaptive inverse control strategy. The research respectively conduct the self-closed-loop test and field test of relevant control strategy to carry on the experiment, the experimental results showed that: in the process of random shock vibration test, by using the filter-X of frequency domain LMS adaptive inverse control strategy implementation on the system impedance matrix of the adaptive correction, effectively restrain noise interference in the system to test the influence of the system control and improve control accuracy; Further, the test can realize the high precision control by using the $\mathrm{X}$-filter of frequency domain LMS adaptive inverse control strategy, thus proving the effectiveness of the proposed control strategy.

\section{References}

1. Ammanagi S, Poornima V, Sera A, et al. Development of a digitally-controlled three-axis earthquake shake table[J]. Current Science. 190-203(2006)

2. Harman C. Multi-axis vibration reduces test time[J]. EE Evaluation engineering. 44-47(2006)

3. Yugang Zhao. Shock response analysis method and its Application[D]. Zhejiang University. (2004)

4. Schoukens J, Rolain Y, Pintelon R. Leakage reduction in frequency-response function measurements[J]. IEEE Transactions on Instrumentation and Measurement. 2286-2291(2006)

5. Boroujeny F B, Chan K S. Analysis of the frequency-domain block LMS algorithm[J]. IEEE Transactions on Signal Processing. 48(2000)

6. Huijun $\mathrm{Yu}$, Zhangwei Chen. Research on adaptive control used for time domain replication of road simulation[J]. Journal of Vibration Engineering. 498-501(2007)

7. Qiangli Luan. Research of key technologies of the control system in hydraulic vibration tests[D]. Zhejiang University. (2015) 